\title{
Antibody Concentration Measurement Using Optical Rotation: Toward Process Analytical Technology
}

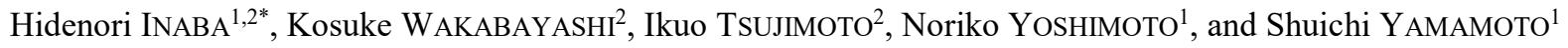 \\ ${ }^{1}$ Graduate School of Medicine, Biomedical Engineering Center, Yamaguchi University, Tokiwadai, Ube-shi, Yamaguchi 755-8611, \\ Japan \\ ${ }^{2}$ Astellas Pharma Inc., 5-2-3, Tokodai, Tsukuba, Ibaraki 300-2698, Japan
}

\begin{abstract}
The antibody manufacturing process consists of the cell culture process using animal cells and the purification process including several chromatography steps and filtration steps. The purification process is important to obtain high quality bio-pharmaceutical products by removing impurities, and measurement of antibody concentrations is a fundamental and essential factor to control the manufacturing process and to confirm the yield of each purification step. Here we introduce the optical rotation method as a resolution for measuring antibody concentrations in the manufacturing process. Feasibility evaluation showed that optical rotation is applicable for measuring a wide range of antibody concentrations even in cell culture supernatants containing various contaminants. In addition, as examples of application to the process analytical technology, we show the possibility of the optical rotation method in the continuous cell culture and chromatography process, and the tangential flow filtration concentration process.
\end{abstract}

\section{Introduction}

The antibody manufacturing process consists of the cell culture process using animal cells and the purification process for removing impurities to obtain high quality bio-pharmaceutical products. The purification process generally includes clarification, chromatography, virus inactivation and virus filtration, and membrane filtration steps. An example of typical antibody purification process is shown in Figure 1.

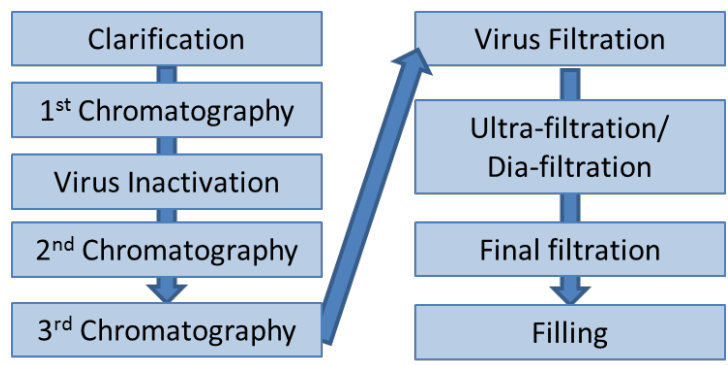

Figure 1. Typical antibody purification process

In the purification process, measurement of antibody concentration is a fundamental and essential factor to control the manufacturing process and to confirm the yield of each purification step. Characteristics of the antibody concentrations include higher concentrations in comparison to the conventional process and a wide concentration range. The antibody concentration in the clarified culture fluid is generally a several $\mathrm{g} / \mathrm{L}$ level, but over $100 \mathrm{~g} / \mathrm{L}$ can be observed for example in the ultrafiltration/ dia-filtration (UF/DF) step.

For concentration measurement, spectrophotometry using UV280 $\mathrm{nm}$ is most common, however, it generally requires sample dilutions due to the limited detection range. Figure 2 shows results of measuring antibody solutions adjusted to various concentrations with a spectrophotometer having an optical path length of $1 \mathrm{~cm}$. In this case, quantitative results of UV280 nm were obtained only in a narrow range of about less than $3 \mathrm{~g} / \mathrm{L}$. In actual manufacturing, dilution of more than 100 times may be required, and multiple dilutions will add much operating time and make a source of analytical errors.

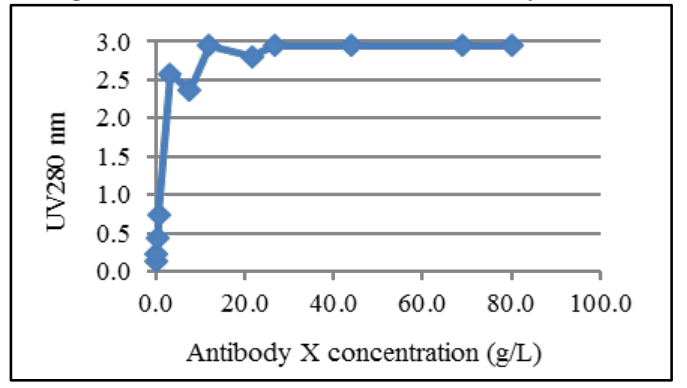

Figure 2. UV280 $\mathrm{nm}$ measurements in antibody $\mathrm{X}$ solutions at various concentrations

To overcome the challenge, a new UV based method without dilution was developed by shortening the optical path length. Moreover, another new method, in which the optical path length changes according to the UV intensity, has been proposed (Anderle and Weber, 2015). However,

\footnotetext{
* Corresponding author: hidenori.inaba $@$ astellas.com
} 
the concentration measurement using UV280 $\mathrm{nm}$ still has disadvantage. UV280 $\mathrm{nm}$ measurement is interfered by impurities having UV absorption. For example, cell culture supernatants in the initial stage of purification process contains not only host cell-derived proteins and DNA but also medium components with UV absorption. Therefore, measurement of antibody concentrations in cell culture supernatant requires different analytical methods such as HPLC, or pre-treatment to remove UV absorbing impurities from the analytical samples.

Optical rotation is a phenomenon in which light rotates in a certain direction when light passes through an optically active substance. It is commonly used to measure purity of sugars and chemical substances, and has already been applied widely as a production control tool in food and chemical manufacturing industries. Meanwhile, it is not used for concentration measurement in the antibody manufacturing process. In addition, there is feasibility for measuring antibody concentrations in cell culture fluid because each substance has its own specific optical rotation. Therefore, we carried out optical rotation measurement for various purification samples and evaluated its practicality.

\section{Experimental}

Optical rotation was measured with a Polarimeter (P2200, JASCO Corporation; Tokyo, Japan). The concentration of a substance contained in a solution can be calculated from the specific optical rotation $[\alpha]$ and the actually measured optical rotation $\alpha$ using the following equation;

$$
\alpha=[\alpha] \times 1 \times \mathrm{c}
$$

where $[\alpha]$ is specific rotation, $\alpha$ is actual rotation, 1 is optical path length, and $\mathrm{c}$ is concentration.

\section{Results and Discussion}

\subsection{Quantitative determination of antibody concentration}

Figures 3 and 4 show relationship between the antibody concentrations and measured optical rotations. Antibody $\mathrm{X}$ and Antibody $\mathrm{Y}$ are different type of antibodies. It should be noted that (1) linearity of quantitative concentration can be obtained in a wide range from the low to high concentrations, (2) it is assumed that further high concentration can be measured because the linearity of optical rotation is still remaining at the highest concentration in this experiment. According to the principal of optical rotation, sugar chains in antibodies should be considered as one reason why good linearity was confirmed between antibody concentrations and optical rotation values. In comparison with the concentration measurement by UV280 nm (Figure 1), the concentration measurement by optical rotation has great advantage in high concentration range analysis.

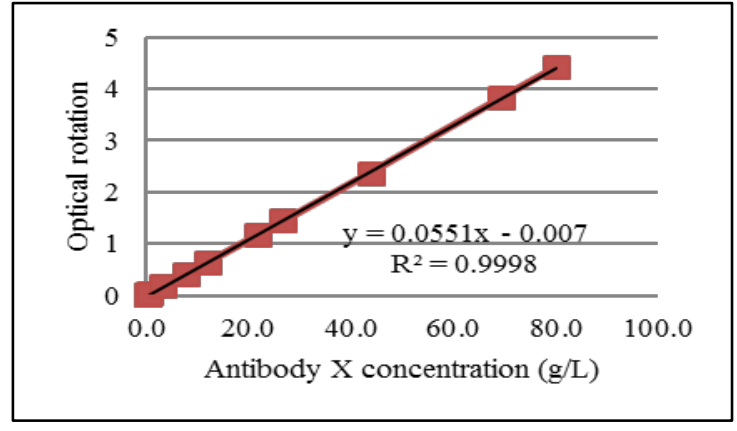

Figure 3. Optical rotation measurement of antibody $\mathrm{X}$ solutions at various concentrations

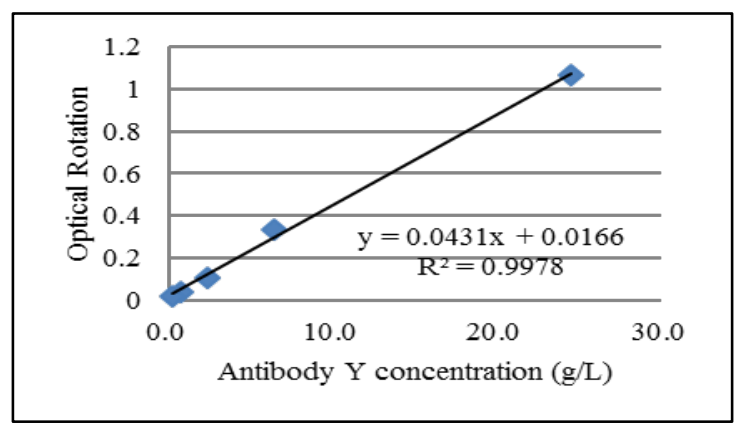

Figure 4. Optical rotation measurement of antibody $\mathrm{Y}$ solutions at various concentrations

\subsection{Accuracy of optical rotation measurement for antibody concentration monitoring}

In order to confirm accuracy of the optical rotation method, linearity, repeatability, and spike and recovery tests were conducted using other antibody. Various concentration samples were prepared, and their concentrations were measured by UV280 $\mathrm{nm}$ and optical rotation. Optical rotation was measured without sample dilution and UV $280 \mathrm{~nm}$ was measured after samples were diluted to adjust the concentrations within the measurable range. As a result, the optical rotation method showed good linearity from low concentrations below $1 \mathrm{~g} / \mathrm{L}$ to high concentrations above $60 \mathrm{~g} / \mathrm{L}$ (Figure 5). With regard to repeatability, optical rotations were measured 5 times per each concentration, and the variation coefficients were calculated. As a result, the variation coefficient tended to increase in the low concentration range, but stable results were confirmed in the medium to high concentration area (Table 1). Furthermore, to evaluate the recovery rate, purified antibody solutions were added to process solutions and the increased concentrations and optical rotations were measured. Recovery rates were calculated by comparing actual increased amounts based on added purified antibody and measured increased amounts based on measured values for each solution. As a result, good recovery rates were obtained as shown in Tables 2 and 3, although the process solutions contain impurities.

As described above, the concentration range in the purification process vary from several $\mathrm{g} / \mathrm{L}$ to over $100 \mathrm{~g} / \mathrm{L}$. The optical rotation method is highly promising as a process monitoring tool based on the linearity, repeatability, and spike and recovery test results. 


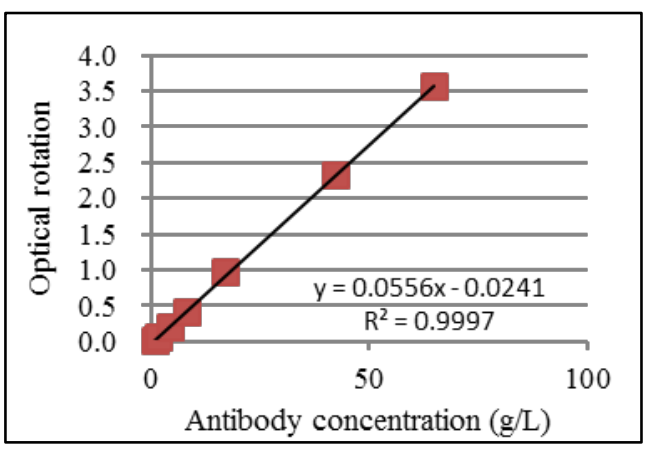

Figure 5. Linearity of optical rotation

Table 1. Repeatability of optical rotation

\begin{tabular}{|c|l|c|c|c|}
\hline \multicolumn{2}{|c|}{} & \multicolumn{3}{|c|}{ Antibody concentration $(\mathrm{g} / \mathrm{L})$} \\
\cline { 2 - 5 } \multicolumn{1}{|c|}{} & 0.22 & 1.69 & 17.12 \\
\hline \hline \multirow{5}{*}{$\begin{array}{l}\text { Optical } \\
\text { rotation }\end{array}$} & $\mathrm{N}=1$ & -0.0150 & 0.070 & 3.555 \\
\cline { 2 - 5 } & $\mathrm{N}=2$ & -0.0135 & 0.074 & 3.573 \\
\cline { 2 - 5 } & $\mathrm{N}=3$ & -0.0107 & 0.073 & 3.555 \\
\cline { 2 - 5 } & $\mathrm{N}=4$ & -0.0131 & 0.072 & 3.552 \\
\cline { 2 - 5 } & $\mathrm{N}=5$ & -0.0093 & 0.072 & 3.572 \\
\cline { 2 - 5 } & Average & -0.0129 & 0.072 & 3.575 \\
\cline { 2 - 5 } & SD & -0.0033 & 0.001 & 0.005 \\
\cline { 2 - 5 } & Variation & & & \\
& coefficient & 25.6 & 2.1 & 0.2 \\
\hline
\end{tabular}

Table 2. Results of spike and recovery test (UV280 nm conc.)

\begin{tabular}{|l|c|c|c|}
\hline & $\begin{array}{c}\text { Conc. } \\
(\mathrm{g} / \mathrm{L})\end{array}$ & $\begin{array}{c}\text { Spiked } \\
\text { conc. }(\mathrm{g} / \mathrm{L})\end{array}$ & $\begin{array}{c}\text { Recovery } \\
(\%)\end{array}$ \\
\hline $1^{\text {st }}$ Chromatography & 7.76 & 12.73 & 102.1 \\
\hline $2^{\text {nd }}$ Chromatography & 7.19 & 12.27 & 101.1 \\
\hline $3^{\text {rd }}$ Chromatography & 5.95 & 11.06 & 102.2 \\
\hline
\end{tabular}

Table 3. Results of spike and recovery test (Optical rotation)

\begin{tabular}{|l|c|c|c|}
\hline & $\begin{array}{c}\text { Optical } \\
\text { rotation }\end{array}$ & $\begin{array}{c}\text { Spiked } \\
\text { optical } \\
\text { rotation }\end{array}$ & $\begin{array}{c}\text { Recovery } \\
(\%)\end{array}$ \\
\hline $1^{\text {st }}$ Chromatography & -0.422 & -0.718 & 98.7 \\
\hline $2^{\text {nd }}$ Chromatography & -0.387 & -0.686 & 98.7 \\
\hline $3^{\text {rd }}$ Chromatography & -0.313 & -0.616 & 99.0 \\
\hline
\end{tabular}

\subsection{Application to process analytical technology}

Process Analytical Technology (PAT) is a system that designs, analyzes, and manages the manufacturing process by real-time monitoring (Holzberg et al., 2018). In order to conduct measurements in real time, PAT must be incorporated into the manufacturing line (in-line), or can be conducted immediately after sampling of process solutions near the manufacturing line (at-line/on-line).

Concentration measurement with the optical rotation detector took only 6 seconds at the shortest in this study. Therefore, it can be considered that the optical rotation is applicable to PAT if the optical rotation detector is incorporated into the manufacturing line using such as a flow cell. Following sections introduce two topics as examples of PAT applications of optical rotation.

\subsection{Application to decide load amount of cell culture supernatant}

Antibody concentrations in a cell culture supernatant cannot be measured with the UV $280 \mathrm{~nm}$ method due to influence of contaminating impurities. In this study, we tried to measure the antibody concentration in the cell culture process by the optical rotation method. As a result, the antibody concentrations measured by the optical rotation method were slightly higher than the actual trend in the early stage of the cell culture process, but good correlation was shown between optical rotations and antibody concentrations in the late stage of the cell culture process (Figure 6). Optical rotation is not influenced by color of cell culture fluid such as UV $280 \mathrm{~nm}$ and can directly related to the amount of asymmetric carbons. In addition, impurity amount was relatively decreased by increasing antibody amount in late stage. Therefore, antibody concentration could be measured using optical rotation in the late stage of cell culture process.

As mentioned above, antibody concentrations in a cell culture supernatant cannot be measured without pretreatment to remove UV absorbing contaminants or using HPLC. On the other hand, the optical rotation method can measure the concentration directly without a dilution step, and the results can be obtained semi-continuously. Therefore, if the optical rotation method is applied to manufacturing process as PAT, it has great advantage to start next step immediately based on the measured antibody concentration.

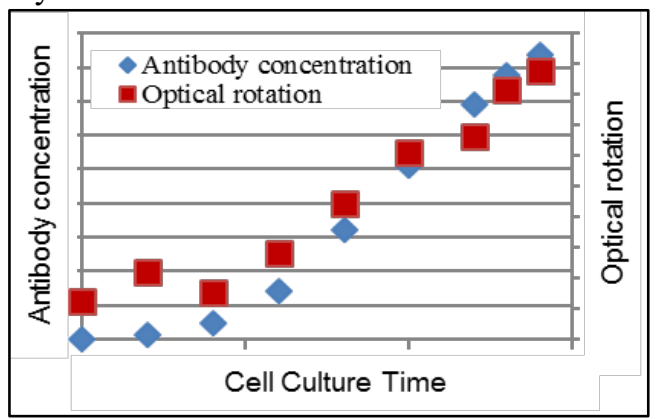

Figure 6. Optical rotation measurements and antibody concentrations in cell culture process

In recent years, continuous manufacturing of biopharmaceuticals has attracted huge attention, and is studied actively all over the world (Arnold et al., 2018). In the continuous process, the first chromatography step should be combined to a perfusion cell culture process, and the antibody concentration of the cell culture supernatant needs to be measured in real time to determine the loading amount onto the first chromatography step.

Based on the result in Figure 6, we consider it is feasible that the first chromatography step combined with the perfusion cell culture process can work continuously by designing the system as shown in Figure 7 . In this 
system, the perfusion culture supernatant passes the optical rotation detector installed into the manufacturing line, and the concentration is measured by real-time monitoring. If the liquid volume of the cell culture supernatant passed through the optical rotation detector is measured using a liquid volume meter, the antibody amount to be loaded onto the next chromatography step can be calculated in real time by multiplying the concentration and the liquid volume. After passing the predetermined loading amount to one of the columns, the flow path is switched to the other column. As a result, continuous processing of the first chromatography step can be realized by alternately repeating the load, wash, elution, and regeneration steps.

This system is expected to be realized, because the antibody concentration can be measured with the optical rotation even in cell culture supernatants containing UV absorbing contaminants, and the measurement results can be obtained in real time by in-line monitoring.

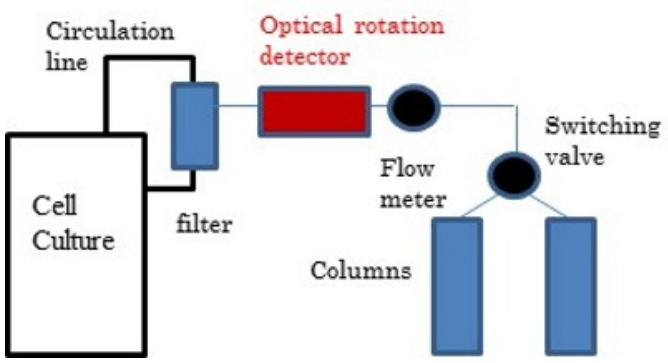

Figure 7. Continuous process design of perfusion culture to first chromatography step

\subsection{Application to decide the endpoint of concentration process}

Antibodies are generally concentrated on the tangential flow filtration (TFF) system using UF membranes. In the TFF step, samples collected from the concentration tank are diluted for measuring the concentration with UV280 $\mathrm{nm}$, or otherwise only UV absorbance in permeate is detected. Real-time monitoring is preferable to improve operability and reduce processing time, and it is highly feasible by application of the optical rotation method to the system as described in Figure 8. If the concentration can be measured continuously, the endpoint of the concentration step can be easily determined in a shorter time without sampling, dilution, or off-line analysis.

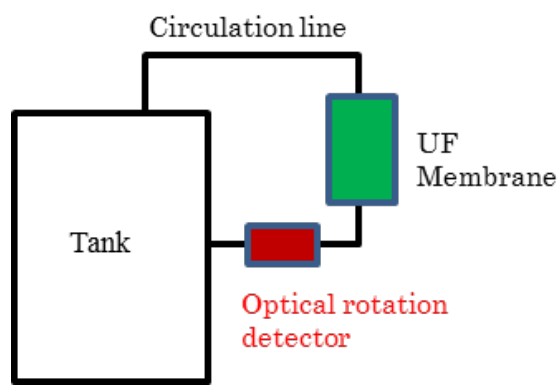

Figure 8. Real-time monitoring design for TFF concentration process

The system shown in Figure 8 was established in the laboratory, and actual experiment was conducted in the small scale model. The result of real-time monitoring of optical rotation is shown in Figure 9. Good correlation was shown between the optical rotations and antibody concentrations by UV280 $\mathrm{nm}$ in the wide concentration range, and the maximum concentration was over $70 \mathrm{~g} / \mathrm{L}$. Based on the experimental result, it should be considered that the optical rotation method can be installed as a PAT technology to the TFF concentration process.

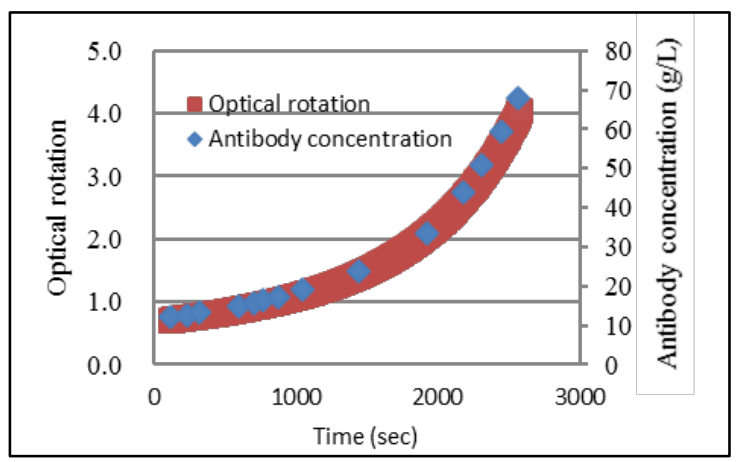

Figure 9. Real-time measurement results of optical rotation in TFF concentration process

\section{Conclusion}

In the antibody purification process, the concentration measurement is a fundamental and essential factor to control the purification process. In this paper, we introduced the optical rotation measurement as a resolution for measuring antibody concentrations in the manufacturing process. The feasibility evaluation showed that optical rotation is applicable for measuring a wide range of antibody concentrations even in cell culture supernatants containing various contaminants. In addition, as examples of application to PAT, we showed the possibility of the optical rotation method in the continuous cell culture and chromatography process and the TFF concentration process.

\section{Acknowledgements}

We would like to thank Dr. Kazutoshi Ban, Mr. Mitsutaka Matsuura, Ms. Azumi Takahashi, and Mr. Kenichi Iwadate for their discussions and input into this manuscript.

\section{References}

Anderle, H. and A. Weber; "Variable Pathlength FiberOptic Spectrophotometry for Protein Determination in Immunoglobulin Concentrates," BioPharm. Int., 28, 4250 (2015)

Holzberg, T. R., V. Watson, S. Brown, A. Andar, X. Ge, Y. Kostov L. Tolosa, and G. Rao; "Sensors for Biomanufacturing Process Development: Facilitating the Shift from Batch to Continuous Manufacturing," Curr. Opin. Chem. Eng., 22, 115-127 (2018)

Arnold, L., K. Lee, J. Rucker-Pezzini, and J. H. Lee; "Implementation of Fully Integrated Continuous Antibody Processing: Effects on Productivity and COGm," Biotechnol. J., 1800061 (2018) 